\title{
Efficient Handover Scheme for Mobile IPv4 over IEEE 802.11 Networks with IEEE 802.21 Triggers ${ }^{1}$
}

\author{
Przemysław Machań, Józef Woźniak
}

Gdańsk University of Technology, Faculty of Electronics, Telecommunication and Informatics, Narutowicza 11/12, Gdańsk, Poland

przemac@o2.pl,jowoz@eti.pg.gda.pl

\begin{abstract}
Handover performance is an important factor for wireless networks, especially nowadays, when multimedia services are becoming increasingly available over the wireless devices. However, users expect uncompromised mobility when using the service. Thus, the support of multimedia services is not possible if handover is inefficient. At the same time it is clear that a strict separation between IP Layer and the Link Layer results in built-in sources of delay. The paper discusses the IEEE 802.11 and Mobile IPv4 handover performance in practical scenarios. We introduce a new simultaneous handover scheme with IEEE 802.21 triggers. In order to verify the handover performance, simulation experiments have been conducted, whose results are also presented and discussed.
\end{abstract}

\section{Introduction}

With the growing speed of wireless networks, multimedia services are becoming increasingly available for mobile users. Wireless devices expect service continuity even when they move between points of attachment. Handover performance is a crucial factor for multimedia services support. These types of services are very sensitive to the channel disruption, handover delays or packet losses. All these factors will significantly lower the quality of multimedia services. Because of this, it is not possible to support multimedia services without fast enough and transparent handover procedures.

The network layer protocol - Mobile IPv4 (MIPv4) was designed without any assumptions about the link layer operation and that has negative implications on handover delay. The strict separation between IP Layer and Link Layer (according to the principles of layered architectures design) results in built-in sources of delay. The first reason is that Mobile Host $(\mathrm{MH})$ can only exchange messages with a directly connected Foreign Agent. In consequence the $\mathrm{MH}$ cannot communicate

1 The work was supported in part by Polish National Center for Research and Development under project PBZ-MNiSW-02/II/2007. 
with a new FA until layer 2 handover is completed. There are two sources of delay: layer 2 handover and event propagation latency to the IP layer. The second one mainly consists of the Mobile IPv4 Registration process latency. During this period the $\mathrm{MH}$ is unable to send or receive any IPv4 packets [8].

The paper is structured as follows. The next section reviews the related work. The following sections describe handover performance in both IEEE 802.11 and MIPv4. Then the IEEE $802.21 \mathrm{draft}$ is discussed. The description of simultaneous handover procedures for layer 2 and layer 3 is subsequently presented. Finally, simulation test-bed is described and results of simulation experiments are presented and discussed.

\section{Related Work}

There is a large number of MIPv4 handover architectures proposed in the literature. One of the most matured extensions to MIPv4 is Low Latency Handoff (LLH) for Mobile IPv4, described in [7]. There are three techniques presented, however the last one is combination of two previous techniques. Pre-Registration handover method allows $\mathrm{MH}$ to prepare its registration state in a new Foreign Agent (nFA) via the old Foreign Agent (oFA), before layer 2 handover commences. The second method is a network-assisted handover that can be either network-initiated or mobile-initiated. Link layer triggers are used on both $\mathrm{MH}$ and FA to invoke particular handover events.

Fast Handover for Mobile IPv4 (FMIPv4) is an adaptation of the Fast Handover for Mobile IPv6. The intention is to utilize the same design for IPv4 networks, however new packet formats for MIPv4 should be standardized. The main idea behind Fast Handover is to obtain a new Care-of Address (CoA) prior to carrying out the handover, and start to use this address just after layer 2 handover is completed. The tunnel is established between the old Access Router (oAR) and the new Access Router (nAR) to enable $\mathrm{MH}$ to send and receive data while the handover proceeds. The main assumptions about network architecture, for Fast Handover, are related to layer 2 and layer 3 interactions. The Access Router must be able to extract the IPv4 address of the nAR from the layer 2 address of the new Access Point (nAP). Similarly to LLH, MIPv6 Fast Handover stack receives a layer 2 trigger when a nAP is discovered.

Both methods assume tightly coupling of layer 2 and layer 3 protocols. Using Pre-Registration protocol from LLH is questionable with IEEE 802.11 as the scanning phase prevents $\mathrm{MH}$ from selecting the new Access Point (nAP) without leaving our current point of attachment [2]. On the other hand, establishing the tunnel between oAP and nAP delays the MIP registration. When the layer 2 handover is completed, the $\mathrm{MH}$ remains registered with the oFA. However, packets destined to the $\mathrm{MH}$ arrive at the oFA, are tunneled to the nFA and are delivered through the nAP.

Both LLH and FMIPv4 are strongly dependent on unspecified layer 2 trigger when handover begins. This trigger cannot be trustworthy in IEEE 802.11 net- 
works as handover detection is the protocol bottleneck and can take more than one second. This delay can lead to a situation when MH looses its connection with oAP before the Pre-Registration procedure is completed.

The simultaneous handover for Mobile IPv4 over IEEE 802.11 (SMIPv4) was originally proposed in [3]. The author suggested extending the IEEE 802.11 specification with the MIPv4-Registration-Request (MIPv4-Reg-Req) Information Element (IE) that can be conveyed in IEEE 802.11 Association Request or IEEE 802.11 Reassociation Request frames. As the described procedure adheres to both Association and Reassociation frames we will use the (Re)Association name to refer to both cases. The IE is extracted by nAP and sent to the nFA as Registration Request. When Registration Response is received at nAP it is compacted into MIPv4-Registration-Reply (MIPv4-Reg-Repl) IE and send back to the MH along with $(\mathrm{Re})$ Association Response message. The new Information Elements have the same fields as MIPv4 Registration related messages.

There are some architectural implications related to the proposed solution. Mobile Host MIP layer must be able to pass its parameters to MAC layer on request. Layer 2 must be able to construct MIPv4 Information Elements. Mobile Host puts its Home Address, as the source address, in the MIPv4-Req-Req IE. The destination address is the multicast address of Mobile-Agents, as defined in MIPv4 specification [13].

IEEE 802.11 Access Point must be able to extract MIPv4 IEs and send them to nFA. If the nFA is co-located with nAP, the MAC and MIP must be able to exchange MIPv4 IEs. If nAP and nFA functionalities are separated nAP operates as proxy for MH. In this paper we will concentrate on the co-located model.

The authors of [10] optimized the simultaneous handover scheme by allowing the nAP to respond to the Association Request message without waiting on MIPv4 Registration Response. This will eliminate the need for MIPv4-Reg-Repl IE and avoid association timer expiration in $\mathrm{MH}$.

The simultaneous handover scheme has a strong advantage over LLH and FMIPv4 solutions. We will propose the extended solution based on standard layer 2 handover end trigger. The advantage of handover end trigger over the handover begin trigger is that the first one can be determined with a high confidence. Although simultaneous handover procedure has strong architectural dependencies, being the clear and tight coupling of layer 2 and layer 3, it is a necessary compromise for an efficient handover. Moreover, because MIPv4 devices are becoming more and more popular, one can expect IEEE 802.11 and MIPv4 solutions to be available on a "single chip" [6].

\section{IEEE 802.11 Handover}

The handover process has the following phases: detection, search, authentication and association. The handover delay can be expressed by formula (1).

$$
\mathrm{T}_{802.11}=\mathrm{T}_{802.11 \text {-detect }}+\mathrm{T}_{802.11 \text {-scan }}+\mathrm{T}_{802.11 \text {-auth }}+\mathrm{T}_{802.11 \text { (r)assoc }}
$$


The detection phase is the time needed for $\mathrm{MH}$ to determine when handover must be performed. During this period the network connection can deteriorate or become unavailable. When the network configuration forces the $\mathrm{MH}$ to change the AP before the channel condition deteriorates the detection time will not affect handover delay. However, network configuration is not always optimized for handover performance. IEEE 802.11 standard does not provide a shared control channel for this information distribution, so the client must scan channels for prospective APs. The next step is the handover execution: authentication and association; these procedures are defined in the IEEE 802.11 standard. If stations support the IEEE 802.11e extension the handover can be delayed with QoS messages. Moreover, if WPA or WPA2 procedures are in use the key derivation and exchange messages will additionally influence the handover delay.

The described delays differ between implementations and depend on network equipment interoperability and environment conditions [17]. Empirical studies were conducted to estimate the values of parameters [9] [14] [18]; the corresponding data was collected and is shown in Table 1.

Table 1. IEEE 802.11 Handover procedure delays

\begin{tabular}{cc}
\hline Parameter & Value \\
\hline $\mathrm{T}_{802.11 \text {-detect }}$ & $300-600 \mathrm{~ms}$ \\
$\mathrm{~T}_{802.11 \text {-scan }}$ & $58-400 \mathrm{~ms}$ \\
$\mathrm{~T}_{802.11 \text {-open-auth }}$ & Less then $10 \mathrm{~ms}$ \\
$\mathrm{~T}_{802.11 \text {-(re)assoc }}$ & Less then $10 \mathrm{~ms}$ \\
\hline
\end{tabular}

The detection phase delay differs when handover is station-initiated or network-initiated. The AP can initiate handover by sending IEEE 802.11 Deassociation Request message. However, in a typical case the station decides to handover when transmission conditions deteriorate. For station-initiated handover the length of detection phase depends strongly on station algorithm.

The explanation for the maximum detection time presented in Table 1 is as follows. If the transmission fails the station assumes collision and retransmits packet at a lower data rate. If the transmission remains unsuccessful, the station assumes signal fading and sends IEEE 802.11 Probe Request to verify the link state. After several unanswered requests the station starts scanning phase.

Generally, there are two groups of detection algorithms, based on: either failed transmissions or received signal strength reported by PHY layer [18]. An example of the algorithm that belongs to the first class is a case when station detects a loss of the connection with an old Access Point (oAP) after three subsequent frames are not sent successfully. In this case $T_{\text {detect }}$ refers to a time needed to send three frames. If the station only receives data or does not send or receive data at all, it can monitor reception of IEEE 802.11 Beacon frames. As typical Beacon frame interval is $100 \mathrm{~ms}$ the detection time can be estimated as $300 \mathrm{~ms}$. 
The algorithms based on signal strength utilized Received Signal Strength Indicator (RSSI) provided by PHY layer - as defined in IEEE specification [4]. The $\mathrm{MH}$ can also use SNR metric. However, a technique to acquire noise level is not covered by the standard. The detection methods based on signal strength typically do not provide the accepted performance because of dynamic nature of wireless channel. Although a number of techniques to shorten detection time is provided in the literature[9][18], this is one of the most important bottlenecks.

The active scanning algorithm is described in IEEE 802.11 standard [4]. This procedure is responsible for a significant part of the handover delay. The station sends Probe Request over a particular channel and waits for either medium busy detection within MinChannelTime or MaxChannelTime timer expiration. The procedure is repeated for each channel to be scanned. However, the standard does not define the timer values and the number of channel to be scanned.

$\mathrm{T}_{802.11 \text {-scan }}=\sum_{\mathrm{c}=1}^{\text {Numchannels }}(1-\mathrm{P}(\mathrm{c})) \cdot \mathrm{T}_{\text {MinchannelTime }}+\mathrm{P}(\mathrm{c}) \cdot \mathrm{T}_{\text {MaxChanneITime }}+\mathrm{T}_{\text {switch }}$

The scanning delay can be represented by equation (2). $\mathrm{T}_{\text {switch }}$ parameter refers to the switch time to a new frequency, resynchronize and start demodulating packets in a new channel. $P(c)$ is the probability that at least one AP will send Probe Response on the selected channel. The described timers are different between implementations and depends on network equipment interoperability and environment conditions.

The number of algorithms is presented in the literature to limit the scanning delay. For example, the authors of SyncScan [14] configure wireless network that the interval between Beacon frames in neighbour channels is constant. According to this scenario Mobile Host can passively scan the next channel in the limited time.

The time for open authentication and reassociation procedures can be modeled as a trivial frame exchange. The measurements show that each procedure takes no more than $10 \mathrm{~ms}$.

\section{MIPv4 Handover}

The MIPv4 handover delay ( $\left.\mathrm{T}_{\mathrm{MIPv}}\right)$ is expressed by equation (3). The delay consists of detection delay, new CoA acquirement and redirection time [15][16].

$\mathrm{T}_{\text {MIPv4 }}=\mathrm{T}_{\text {MIPv4-detect }}+\mathrm{T}_{\text {MIPv4-coa }}+\mathrm{T}_{\text {MIPv4-redirect }}$

The detection time is defined as an interval between the time instance when link layer connection is reestablished with a new AP and the beginning of CoA acquisition procedure. In the next step the station needs to retrieve information about a new care-of-address and the default gateway to resume communication on the new subnet. The time for this procedure is referred to as $T_{\text {MIPv4-coa. }}$ Once the required IP level information is obtained, the station redirects its upstream and 
downstream flows ( $\mathrm{T}_{\text {MIPv4-redirect }}$ ). The timing of MIPv4 handover is presented in Fig. 1.

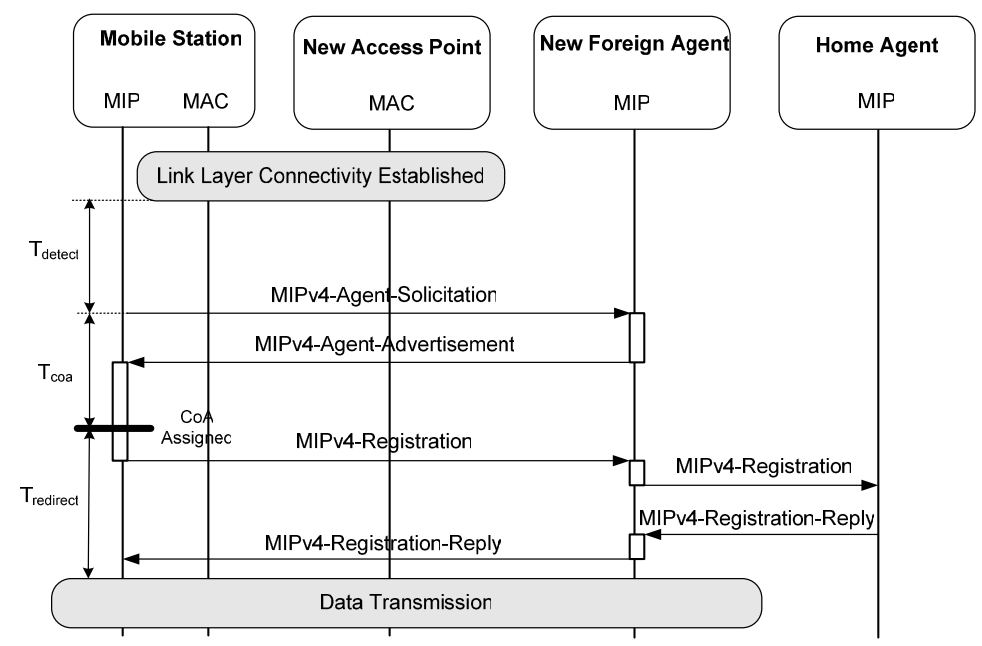

Fig. 1. MIPv4 Handover Timing

To evaluate the handover performance, the more detailed assumptions must be accepted. Detection time depends on the move detection algorithm used. There are three mechanisms proposed for Mobile IPv4 draft: Lazy Cell Switching (LCS), Prefix Matching (PM), and Eager Cell Switching (ECS)[13]. In our experiment the mobile station uses the ECS method. The station records the lifetime received from Agent, with which $\mathrm{MH}$ is currently registered. If the lifetime expires until the next Agent Advertisement (AA) is received the station assumes that connectivity with this Agent is lost and the station should perform CoA retrieval procedure. However, $\mathrm{MH}$ can attempt to register with another agent if Agent Advertisement from $\mathrm{nFA}$ is received before the lifetime of the current Agent expires. Assuming that the advertisement lifetime is $\mathrm{T}_{\mathrm{AD}-\mathrm{LT}}$ and Agent Advertisements period is $\mathrm{T}_{\mathrm{AD}}$ the detection time is presented by equation (4). The assumption behind equation (4) is that layer 2 handover is instant. In fact the MIPv4 detection period begins along with IEEE 802.11 handover process, but the first is typically longer.

$\mathrm{T}_{\text {MIPv4-detect(LCS) }}=\frac{\min \left(\mathrm{T}_{\mathrm{AD}-\mathrm{LT}}, \mathrm{T}_{\mathrm{AD}}\right)}{2}-\frac{\min \left(\mathrm{T}_{\mathrm{AD}-\mathrm{LT}}, \mathrm{T}_{\mathrm{AD}}\right)^{3}}{6 \mathrm{~T}_{\mathrm{AD}-\mathrm{LT}} \mathrm{T}_{\mathrm{AD}}}$

The evaluation of detection time depends directly on Agent Advertisement period. Advertisement lifetime should be at least three times higher then AA period. The AA rate was initially limited to one per second [13] to save the wireless bandwidth. However, with the increasing wireless network speed and the demand for seamless handover, the AA period can be lowered. The authors assumed AA period to be one second. Both $\mathrm{T}_{\text {MIPv4-coa }}$ and $\mathrm{T}_{\text {MIPv4-redirect }}$ can be modeled as frame 
exchange and not introduce a significant delay to the MIPv4 handover procedure. The handover phase delays are collected in Table 2.

Table 2. MIPv4 Handover procedure delays

\begin{tabular}{cc}
\hline Parameter & Value \\
\hline $\mathrm{T}_{\text {MIPv4-detect(LCS) }}$ & $100-1000 \mathrm{~ms}$ \\
$\mathrm{~T}_{\text {MIPv4-coa }}$ & Less then $10 \mathrm{~ms}$ \\
$\mathrm{~T}_{\text {MIPv4-redirect }}$ & Less then $40 \mathrm{~ms}$ \\
\hline
\end{tabular}

\section{IEEE 802.21 Framework}

The IEEE 802.21 standard introduces Media Independent Handover (MIH) Function that is considered a shim layer in the network stack of both network node and the network elements that provide mobility support [5]. MIH Function provides abstracted services to the upper layers and communicates with lower layers through technology-specific interfaces. Handover control, handover polices and other algorithms involved in handover decision-making are handled by communication system elements and are not part of the IEEE 802.21 specification.

The scope of IEEE 802.21 standard will include a universal architecture that provides service continuity while a $\mathrm{MN}$ switches between heterogeneous linklayer technologies. The MIH Function provides the following services: Media Independent Event Service (MIES), Media Independent Command Service (MICS), and Media Independent Information Service (MIIS).

MIES provides both local and remote events and triggers to the upper layers of MN. Typical events are MIH Link Up or MIH Link Parameters Change, originated in layer 2. MICS provides functions to gather the status of links and invoke commands to control handover process. The commands receiver can be both local and remote. Typical commands are MIH Poll used to poll physical links or MIH Configure used to configure connected links. MIIS defines access to network database that contains information used to aim handover process. The network information is stored in platform independent description language and can be: static and dynamic. Static information examples are network and provider name, whilst dynamic information comprises a channel, security configuration and MAC addresses.

\section{Simultaneous Handover with IEEE 802.21 triggers}

The concept of simultaneous handover assumes tight coupling of layers 2 and 3 protocols that should result in improved handover efficiency. In the paper we extend the procedure proposed in [10] by the usage of the standard MIH Link Up trigger. This will make it possible to simplify the implementation of protocol on MH. MIPv4 instance does not need to pass parameters to MAC layer and can operate transparently. The handover procedure is depicted in Fig. 2. 
The handover procedure begins when Mobile Host MAC detects the handover that is marked as L1 trigger. The active scanning procedure is invoked in the next step. When nAP is selected the Link Up event is passed to the MIH layer and propagated to the MIH Client (MIP). When the event is received the Mobile IPv4 layer sends the Registration Request message. The RR message is transformed into Registration-Request-IE in the MAC layer. The other operations are the same as in the base protocol.

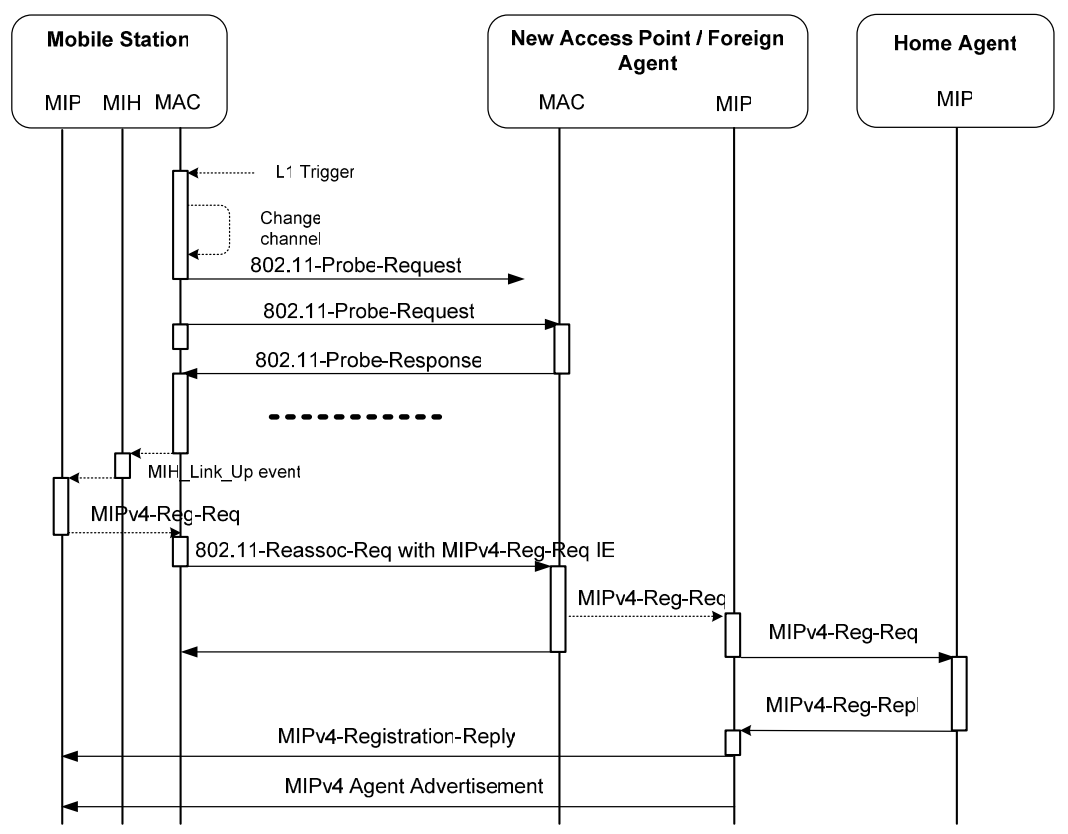

Fig. 2. Simultaneous handoff for IEEE 802.11 and MIPv4

\section{Test-bed Implementation}

The model of simultaneous handover with our extension was implemented in widely used ns-2 simulator. We based on handover support developed for Seamless and Secure Mobility project [12]. For the purpose of the simulation the model of a "city market" was created, as presented in Fig. 3. There are three Mobile Routers (MR), each of them have both IEEE 802.11 Access Point and MIPv4 Foreign Agent functionalities. The stations move within an area of 180 x 60 meters with the velocity of $1 \mathrm{~m} / \mathrm{s}$. The number of stations and traffic load was changed to verify correctness of the protocol operation. The experiments were conducted using 10 different, random mobility patterns.

Mobile stations were downloading CBR stream using 1000-bytes-long fixedsize UDP packets. The reason for using UDP, and not TCP, is that TCP infers congestion from packet loss and scales back its send window accordingly. The ex- 
periments aimed at how throughput, handover delay and packet loss are affected by handover algorithms, rather than due to protocol-induced throughput reductions. Although TCP is used for many network applications, the majority of realtime multimedia services are based on UDP.

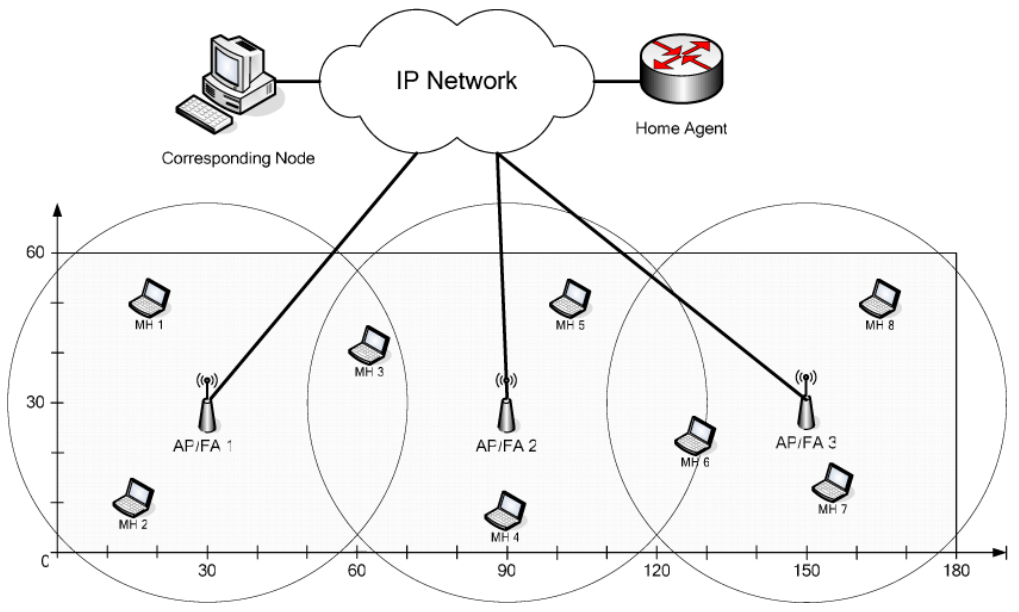

Fig. 3. Simulation scenario for simultaneous handover

\section{Simulation Results}

The experiments were conducted to compare the handover performance using original and simultaneous handover procedures. The handover delay measurements are presented in Fig. 4. Station number in the experiments is marked in the legend as $n$. The delay was measured as time between last packet received by MH through the old MR and the first packet received via the new MR. The handover delay does not depend on the network load or the number of stations, but results were presented for consistency. The regular handover scenario is implemented without any handover-optimized mechanisms. MIPv4 handover proceeds independently of IEEE 802.11 handover. The variation of handover delay for regular handover is higher than using simultaneous handover. The reason is that regular MIPv4 handover time is dependent on $\mathrm{T}_{\mathrm{AD} \text { - } \mathrm{LT}}$ and $\mathrm{T}_{\mathrm{AD}}$ timers as described previously.

The detection delay $\left(\mathrm{T}_{\mathrm{SMIPv} 4-\mathrm{detect}}\right)$ is, in the case of simultaneous handover procedure, the time between the IEEE 802.11 (Re)Association Response message is received by $\mathrm{MH}$ and MIH Link Up trigger is received by MIPv4 layer. The detection delay strongly depends on internal $\mathrm{MH}$ design and its value, in our experiments, was below $1 \mathrm{~ms}$. In turn, the total delay for simultaneous handover $\left(\mathrm{T}_{\mathrm{SMIPv} 4}\right.$ $802.11=550 \mathrm{~ms}$ ) was about $45 \%$ lower when compared with the regular scenario $\left(\mathrm{T}_{\text {MIPv4-802.11 }}=1000 \mathrm{~ms}\right)$. However, the value of $\mathrm{T}_{\mathrm{SMIPv} 4-802.11}$ is still not accepted for multimedia services. Using simultaneous handovers the layer 3 handover delay 
$\mathrm{T}_{\mathrm{SMIPv} 4}$ is optimized to less then $30 \mathrm{~ms}$, compared to $\mathrm{T}_{\mathrm{MIPv} 4}=450 \mathrm{~ms}$ when using the regular protocols. In further investigations we plan to optimize $T_{802.11}$ - the layer 2 handover.

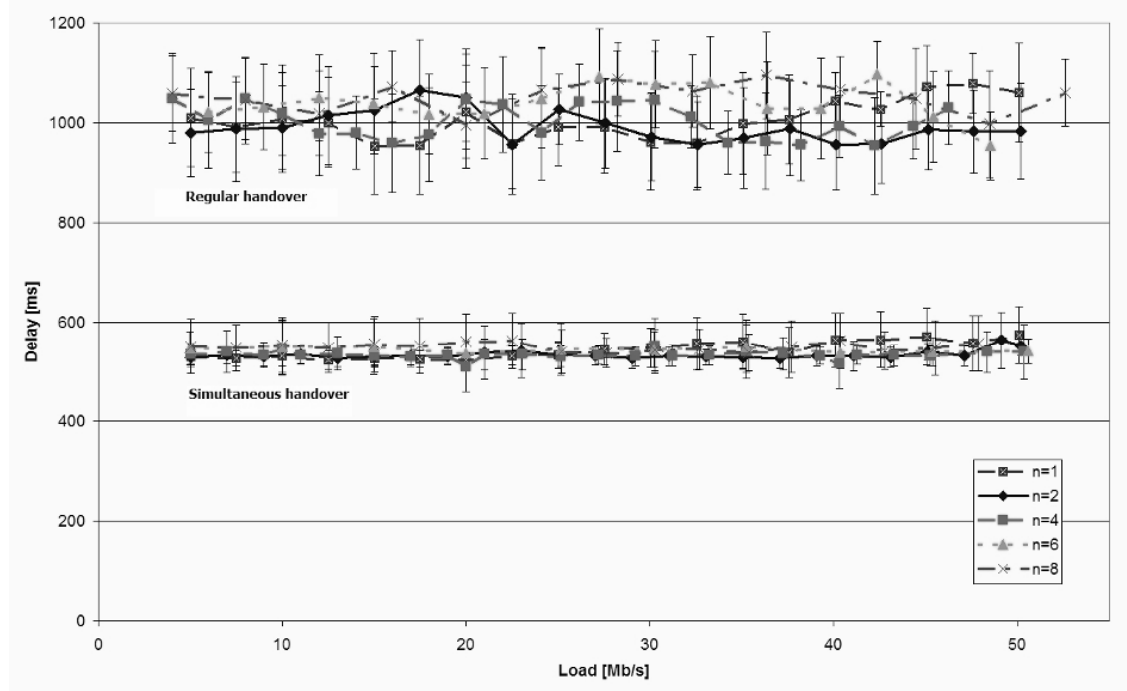

Fig. 4. Handover delay vs. network load

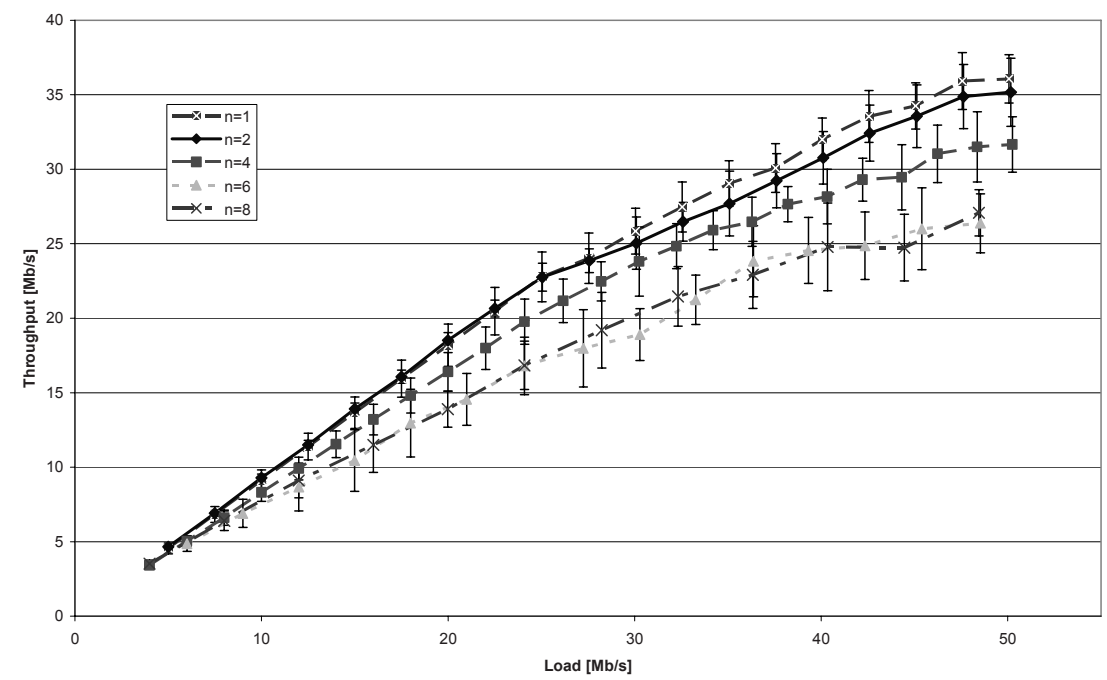

Fig. 5. Data throughput vs. network load in the regular handover scenario

The results of handover delay can be roughly compared with LLH and FMIPv4 performance. The simulation results presented in [1] show that packets sent during 
the handover with LLH or FMIPv4 experience the delay not longer then $100 \mathrm{~ms}$. Although simulation scenarios were different, we can estimate that SMIPv4 handover delay is shorter compared with the LLH and FMIPv4 protocols.

The data throughput variations vs. network load are presented in Fig. 5 and Fig. 6. The charts show only user data; signal and protocol messages (e.g. IEEE 802.11 Management and Control frames, MIPv4 Registration frames) were not measured. The effective throughput of the network with SMIPv4 handover is slightly higher when compared to MIPv4 case because of the shorter handover delays.

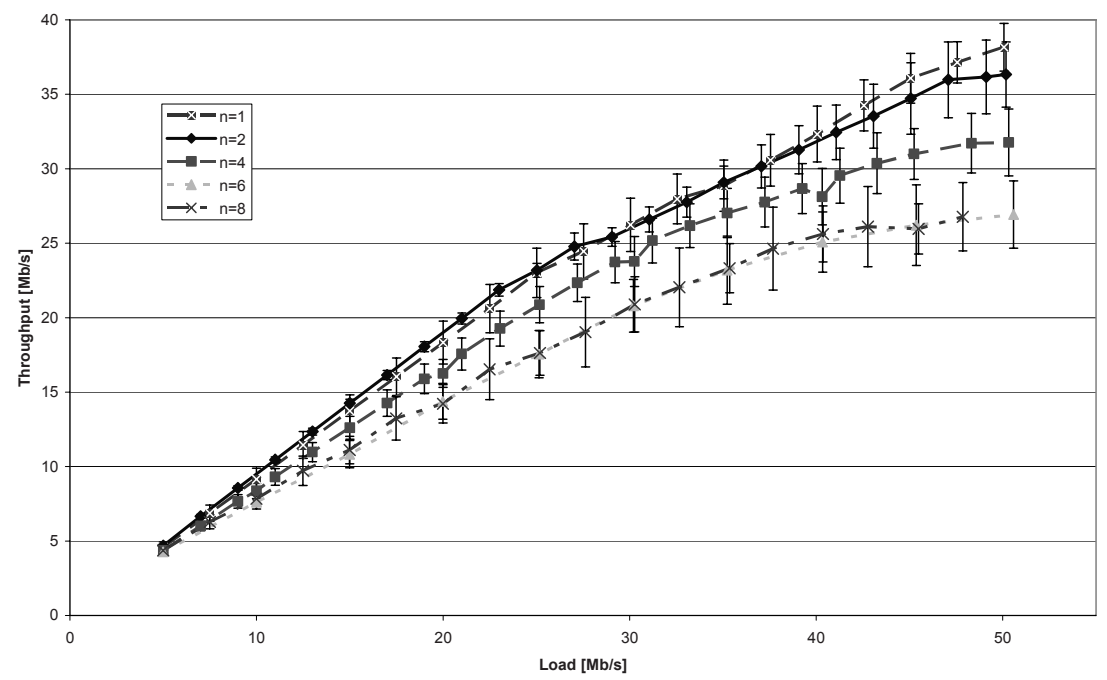

Fig. 6. Data throughput vs. network load in the simultaneous handover scenario

\section{Conclusions}

The article presents a handover performance analysis with respect to the overall delay. The delay components in each layer were selected and described. The main interest was in the MIPv4 protocols performance. Existing protocols that claim to support fast handovers are based on the layer 2 trigger indicating handover begin. This IEEE 802.21 trigger is unreliable when handover is typically station-initiated, as is the case in IEEE 802.11. We have proposed and described the simultaneous handover procedure that uses the layer 2 handover end trigger. The main advantage of our solution over the previously described is that handover end can be trustfully determined in IEEE 802.11 networks. The simultaneous handover protocol was modeled using ns-2. The results show that layer 3 handover was optimized; the total handover delay was shortened from $1050 \mathrm{~ms}$ to $550 \mathrm{~ms}$ in typical scenarios. 


\section{References}

1. Blondia C Casals O Cerdà L Van den Wijngaert N Willems G (2004) Performance Evaluation of Layer 3 Low Latency Handoff Mechanisms. Mobile Netw and Appl 9:633-645

2. De Cleyn P Van den Wijngaert N Cerdà L Blondia C (2004) A Smooth Handoff Scheme Using IEEE802.11 Triggers - Design and Implementation. Comp Netw:345-361

3. Goswami S (2003) Simultaneous Handoff of Mobile-IPv4 and 802.11. Internet Draft

4. IEEE Comp Society (1999) Part11: Wireless LAN Medium Access Control (MAC) and Physical Layer (PHY) Specifications.

5. IEEE Comp Society (2007) P802.21/D05.00: Draft IEEE Standard for Local and Metropolitan Area Networks: Media Independent Handover Services.

6. Love J (2005) Thinking Big: These single-chip switch processors take on enterprise wired/wireless LANs. http:/www.eeproductcenter.com /testmeasure/review/showArticle.jhtml?articleID=60402054. Accessed 24 March 2008

7. Malki El K (ed) (2007) Low-Latency Handoffs in Mobile IPv4. RFC 4881

8. Matusz P, Machan P, Wozniak J (2003) Analysis of profitability of intersystem handovers between IEEE 802.11b and UMTS. IEEE Conf on Local Comput Netw

9. Mishra A Shin M Arbaugh W A (2003) An Empirical Analysis of the IEEE 802.11 MAC Layer Handoff Process. ACM SIGCOMM Comput Commun Rev:93-102

10. Mun Y Park J (2003) Layer 2 Handoff for Mobile-IPv4 with 802.11. Internet Draft

11. Mun Y Park J (2004) The Layer 2 Handoff Scheme for Mobile IP over IEEE 802.11 Wireless LAN. Springer-Verlag LNCS 3043:1144-1150

12. NIST (2007) Seamless and Secure Mobility. http://www.antd.nist.gov/ seamlessandsecure.shtml. Accessed 01 June 2008

13. Perkins C (ed) (2002) IP Mobility Support for IPv4. RFC 3344

14. Ramani I Savage S (2005) SyncScan: Practical Fast Handoff for 802.11 Infrastructure Networks. IEEE Infocom Conf

15. Rückforth T (2004) AAA Context Transfer for Fast Authenticated InterDomain Handover. Swisscom SA Innovations Broadband Network, Bern

16. Vatn J O (2000) Improving Mobile IP handover performance. Royal Institute of Technology, Stockholm

17. Vatn J O (2003) An experimental study of IEEE 802.11b handover performance and its effect on voice traffic. Royal Institute of Technology, Stockholm

18. Velayos H, Karlsson G (2004) Techniques to reduce the IEEE 802.11b handoff time. IEEE Int Conf on Commun 